\title{
Brachial Plexus Continuous Block in Management of Stiff Post Arthroscopy Shoulders
}

\section{Munawar Shah*, Danial Shah, Kishen Parekh, Muhammad Sufyan and Tadas Kananavichius}

Department of T\&O, Manor Teaching Hospital Walsall, UK

*Corresponding Author: Munawar Shah, Department of T\&O, Manor Teaching Hospital Walsall, UK.
Received: November 09, 2020

Published: December 14, 2020

(C) All rights are reserved by Munawar

Shah., et al.

\begin{abstract}
Introduction: After shoulder subacromial decompression (SAD) and Acromioclavicular joint (ACJ) excision when the patient for whatever reason does not mobilize shoulder according to a postoperative protocol becomes stiff and as the pain increases the stiffness worsens and end up in a vicious cycle.

We are presenting our use of continuous brachial plexus block in stiff shoulders following surgery with an acceptable outcome.

Method: A retrospective study was conducted in Walsall Manor Hospital of the patient undergoing Continuous Brachial plexus Block from January 2014 to December 2018 in patients with secondary frozen shoulder following simple post-arthroscopy Sub-acromial decompression (SAD) +/- acromioclavicular joint (ACJ) excision. Patients underwent Physiotherapy and were then followed up in the clinic at 8 weeks. About $90 \%$ were discharged at this stage. However, about 6 weeks further physiotherapy was offered to the strugglers. The Resistant cases were included after being filtered out for disc disease and Local anesthetic complications. All people fulfilling the criteria were listed for continuous brachial plexus block with a catheter for 48 hours along with physiotherapy. Patients followed and reviewed at 6/52 6/12 and 1 year. Constant score (1) was calculated at admission and 1 year follow up. Data analyzed using SPSS 20.0.

Results: 401 shoulder arthroscopy for subacromial decompression and acromioclavicular joint decompression were carried out in a period of 5 years. At 14 weeks follow up post-surgery 28 patients were still struggling with pain and stiffness. Cervical disc disease of C5/C6 has been diagnosed in 5 patients and excluded from the study. Of the 23 patients ended up with being listed for Continuous Brachial plexus block. At 1-year post-block facilitated aggressive physiotherapy (for initial 48 hours) patients had remarkably improved clinical and functional outcomes.

Conclusion: We report that in resistant cases after simple arthroscopy posterior capsule tightness is a cause and a continuous brachial plexus block is an ideal treatment with satisfactory results.
\end{abstract}

Keywords: Shoulder Arthroscopy; Post Arthroscopy stiffness, continuous Brachial plexus block

\section{Introduction}

Subacromial impingement is one of the most common causes of shoulder pain [1]. It is usually nonresponsive to conservative treatment. The range of pathology is from subacromial bursitis to tendinopathy and rotator cuff tears. Arthroscopic Subacromial decompression is the treatment of choice to remove the physical cause of impingement which is usually a curved acromion or ACJ arthritis. The procedure is simple with an excellent outcome [2]. One of the reliable procedures to improvise the functional ability of a patient with sub acromial impingement syndrome is arthroscopic subacromial decompression, increasing constant score at the three-week post-surgical period from 20 points to 40 points. Hence, Patients can be counselled about notable functional improvement within a short period of the surgery [3]. Postoperative range of motion returned to normal in $90 \%$ patients within 6 months [4]. After shoulder decompression and $\mathrm{ACJ}$ resection when the patient for whatever reason do not mobilize shoulder according to a postoperative protocol become stiff and as the pain increases the stiffness wors- 
ens and end up in a vicious cycle [5,6]. There is an incidence of more than 5 percent reported lately for post arthroscopic subacromial decompression and ACJ excision stiffness [7]. We are aware of studies in resistant shoulder pain with a continuous block that has helped $[8,9]$.

We are presenting our use of continuous block in stiff shoulders following simple Shoulder arthroscopic SAD +/- ACJ excision.

\section{Method and Study}

This retrospective study was conducted in Walsall Manor Hospital of patient undergoing Continuous Brachial plexus Block from January 2014 to December 2018 during which 401 patients underwent shoulder arthroscopy with diagnosis of Impingement syndrome and Acromioclavicular arthritis. All patients were day surgery cases and had one shot inter-scalene block for surgery. Post arthroscopy SAD/ACJ patients underwent Physiotherapy and were then followed up in clinic at 8 weeks. About $90 \%$ were discharged at this stage. Whereas, 6 weeks further physiotherapy was offered to the strugglers after a sub acromial injection of Steroid and long acting local anaesthetic. At a total of 14 weeks additional 3\% were discharged. The Resistant cases were filtered for disc disease and the rest listed for continuous brachial plexus block for 48 hours. The Patient was Admitted for Block EUA was done and movements noted followed by Continuous block with measured top up and Intense Physiotherapy. Block stopped at 48 hours and Physiotherapy Continued after withdrawal of catheter for 6/52.Patients followed and reviewed at 6/52, 6/12, and 1 year. Murley Constant score was calculated at admission and at 1 year follow up.

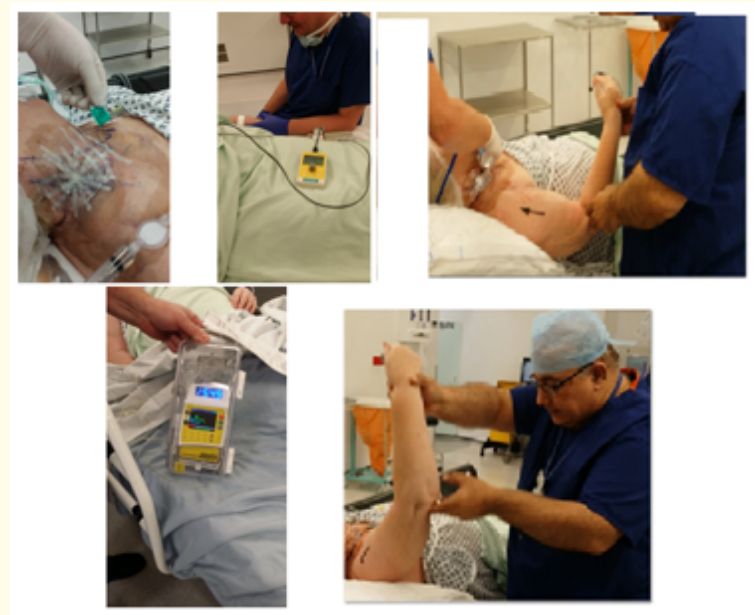

Figure a

Data was entered and analysed using SPSS version 20.0. Mean and standard deviation were calculated for quantitative variable while frequency and percentages for qualitative variables. Normal- ity of data was assessed using Shapiro-wilk test. Post stratification Wilcoxon signed rank test applied to analysed quantitative variables. P-value of $<0.05$ considered as significant.

\section{Results}

401 shoulder arthroscopy for sub acromial decompression and acromioclavicular joint decompression were carried out in a period of 5 years. At 14 weeks follow up post-surgery 28 patients were still struggling with pain and stiffness. Out of which 5 patients had C5/C6 cervical disc disease. Of these 23 patients 15 were Females and 7 males with an average age of 40 (31-47) ended up with being listed for Continuous Brachial plexus block. At 1 year post block 22 patients had excellent outcome 1 patient a female was still symptomatic. There were no reoperations 1 patient needed medication and there were marked improvement in constant scores. One catheter fell out and was redone. There were no symptomatic hemi diaphragm and no Haematomas. There is significant improvement in pain (Table 1), activity level (Figure 1) ROM (Figure 2) and Constant Murley score (Figure 3).

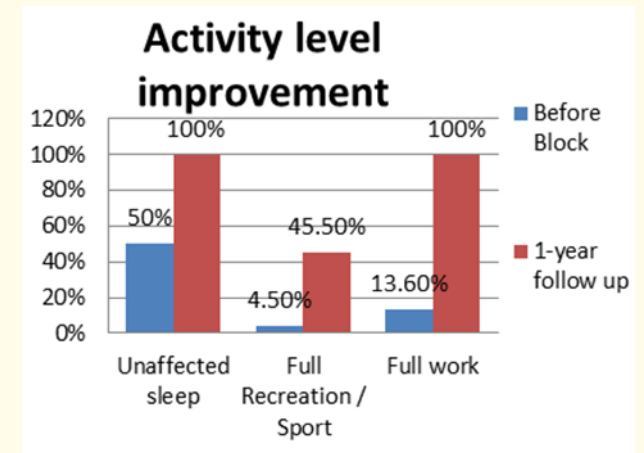

Figure 1

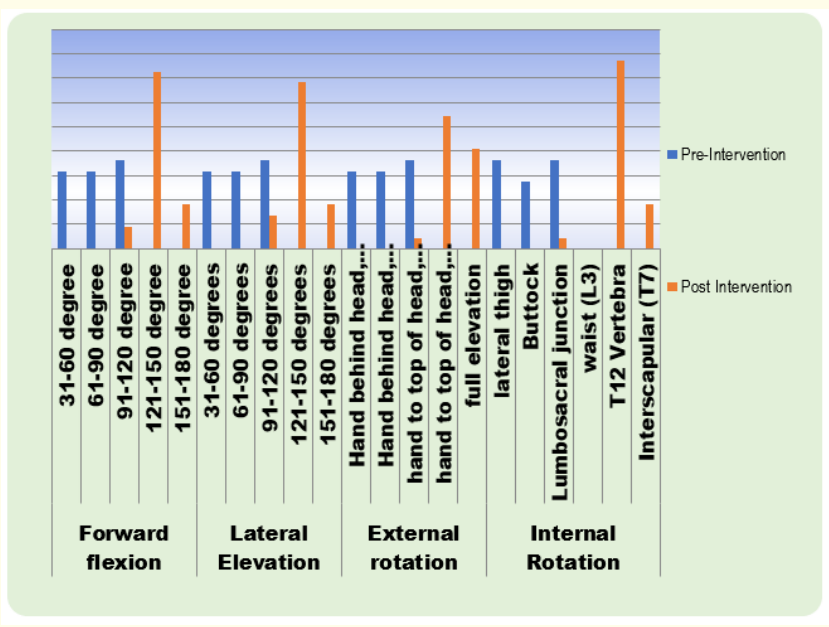

Figure 2: Range of Motion. 


\begin{tabular}{|c|c|c|c|c|}
\hline \multirow{2}{*}{\multicolumn{2}{|c|}{$\begin{array}{c}\text { Variables } \\
(n=22) \\
\text { Mean } \pm \text { SD/Frequency }\end{array}$}} & \multirow{2}{*}{$\begin{array}{c}\text { Pre-Intervention } \\
\text { Mean } \pm \text { SD/Frequency }\end{array}$} & \multirow[t]{2}{*}{$\begin{array}{c}\text { 1-year Follow up } \\
\text { (post-brachial Block) }\end{array}$} & \multirow[t]{2}{*}{ p-value } \\
\hline & & & & \\
\hline \multirow[t]{4}{*}{ Pain } & Severe & $7(31.8 \%)$ & $0(0 \%)$ & \\
\hline & Moderate & $14(63.6 \%)$ & $0(0 \%)$ & \\
\hline & Mild & $1(4.5 \%)$ & $14(63.6 \%)$ & \\
\hline & None & $0(0 \%)$ & $8(36.4 \%)$ & \\
\hline \multirow[t]{5}{*}{ Arm Position } & Up to Waist & $7(31.8 \%)$ & $0(0 \%)$ & \\
\hline & Up to Xiphoid & $2(9.1 \%)$ & $0(0 \%)$ & \\
\hline & Up to Neck & $11(50 \%)$ & $0(0 \%)$ & \\
\hline & $\begin{array}{l}\text { Up to top of } \\
\text { head }\end{array}$ & $2(9.1 \%)$ & $11(50 \%)$ & \\
\hline & Above head & $0(0 \%)$ & $11(50 \%)$ & \\
\hline \multirow{2}{*}{$\begin{array}{l}\text { Abduction } \\
\text { strength }\end{array}$} & 0 & $0(0 \%)$ & $0(0 \%)$ & \\
\hline & $1-3$ & $7(31.8 \%)$ & $0(0 \%)$ & \\
\hline \multirow[t]{8}{*}{ (Pounds) } & $4-6$ & $8(36.4 \%)$ & $0(0 \%)$ & \\
\hline & $7-9$ & $7(31.8 \%)$ & $0(0 \%)$ & \\
\hline & $10-12$ & $0(0 \%)$ & $0(0 \%)$ & \\
\hline & $13-15$ & $0(0 \%)$ & $0(0 \%)$ & \\
\hline & $16-18$ & $0(0 \%)$ & $3(13.6 \%)$ & \\
\hline & $19-21$ & $0(0 \%)$ & $7(31.8 \%)$ & \\
\hline & $22-24$ & $0(0 \%)$ & $12(54.5 \%)$ & \\
\hline & $>24$ & $0(0 \%)$ & $0(0 \%)$ & \\
\hline \multicolumn{2}{|c|}{ Constant-Murley Score* } & $35.14 \pm 16.77$ & $82.86 \pm 10.64$ & $<0.001$ \\
\hline
\end{tabular}

Table 1

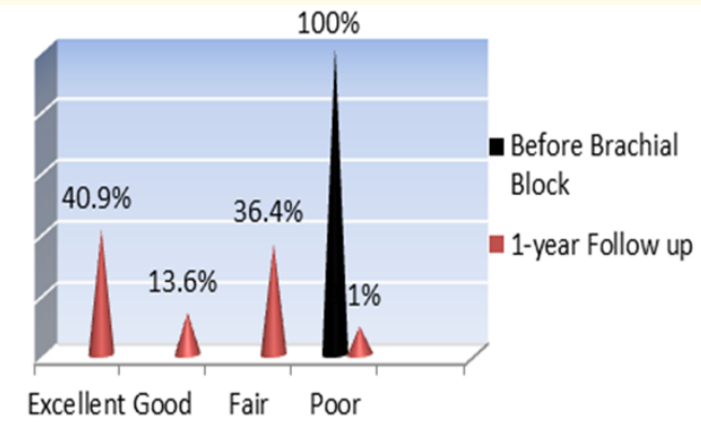

\begin{tabular}{|lc|}
\hline $\begin{array}{l}\text { Grading } \\
\text { (Difference between normal and Abnormal Side) }\end{array}$ & Shoulder Score \\
\hline$>30$ Poor, 21-30Fair, 11-20Good <11Excellent \\
\hline
\end{tabular}

Figure 3

\section{Discussion}

A complete history and examination including skin sensitivity to exclude nerve injury is an essential first step in deciding the root cause of shoulder stiffness as well as planning the best treatment approach for the patient. [10] With a comprehensive history, one can obtain important information on the reasons for the shoulder stiffness, such as failure of compliance to physiotherapy, prolonged immobilization, inadequate pain control/lowered pain threshold, use of improper loads and falls, and other incidences leading to new pathologies [10]. A sub acromial injection of local anaesthetic can help in assessing intensity of pain inhibiting shoulder mobilization. This was also used to exclude and isolate c spine problems as there is a fair amount of grey areas in referred pain [11]. Referred Pain Mapping Blue is Neck, Red is Shoulder and Green is spine. The arm squeeze test was also used but in our hands was not conclusive to diagnose cervical spine problems [12]. Early rehab in form 
of Physiotherapy is important to improve functional recovery following Shoulder Arthroscopy. Pain relief is essential for patients to participate Regional anaesthesia in the form of a peripheral nerve block can provide substantial pain control while also limiting the use of opioid medications especially after stiffness [13]. Similar experience in lower limb Arthroplasty is published [14,15] and also available in complex elbow surgery [6]. Evidence is also present in management of Shoulder Syndrome or CRPS of shoulder [16].

In 1987, the constant-Murley score was introduced as a system to assess shoulder function, irrespective of diagnosis [18]. European Society for Surgery of the Shoulder and Elbow approved and recommended and ever since then has been widely applied as an assessment method [6,17]. The Constant-Murley score contains both physician-completed and patient-reported portions. The four domains include pain (15 possible points), activities of daily living (20 possible points), mobility (40 possible points), and strength (25 possible points). Scores range from 0 points (most disability) to 100 points (least disability) [18].

The current evidence validates and supports the Constant Murley as a gold standard in shoulder evaluation for sub acromial pathology; but data are inconclusive for other shoulder conditions $[15,17]$. As all our patients were post sub acromial surgery we feel that its use is justified.

\section{Conclusion}

We report that in resistant cases after simple arthroscopy procedure, a continuous brachial plexus block (48 hours) with intense physiotherapy is an acceptable and reliable treatment option with satisfactory clinical and functional outcome. It is evident in our study, that use of continuous brachial plexus block for up to 48 hours in stiff shoulders following simple Shoulder arthroscopic SAD +/- ACJ excision, and in-hospital intense physiotherapy has overall improved outcomes. Reduced pain and improved overall activity including sleep and return to work is the essential goals for improved quality of life.

\section{Bibliography}

1. Michener LA., et al. "Anatomical and biomechanical mechanisms of subacromial impingement syndrome". Clinical Biomechanics (Bristol, Avon) 18.5 (2003): 369-379.

2. Ellman H. "Arthroscopic subacromial decompression: Analysis of one- to three-year results". Arthroscopy 3 (1987): 173181.
3. R Dodenhoff. Rate of recovery after arthroscopic subacromial decompression online.boneandjoint.org.uk (2002).

4. Monga P., et al. "Stiffness after Arthroscopic Shoulder Surgery: Incidence, Management and Classification". Shoulder and Elbow 4.3 (2012): 169-173.

5. Hiscock N., et al. "Pain, depression and the postoperative stiff shoulder”. BMC Musculoskeletal Disorder 16 (2015): 376.

6. Vrotsou K., et al. "Constant-Murley Score: systematic review and standardized evaluation in different shoulder pathologies". Quality of Life Research 27 (2018): 2217-2226.

7. Evans J P., et al. "Frozen shoulder after simple arthroscopic shoulder procedures". The Bone and Joint Journal 97 (2015): 963-966.

8. P H K Mak., et al. "Functional improvement after physiotherapy with a continuous infusion of local anaesthetics in patients with complex regional pain syndrome". Acta Anaesthesiologica Scandinavica 47.1 (2003): 94-97.

9. Narinder Rawal., et al. "Patient-controlled Regional Analgesia (PCRA) at Home: Controlled Comparison between Bupivacaine and Ropivacaine Brachial Plexus Analgesia". Anesthesiology 96.6 (2002): 1290-1296.

10. Th Fabre., et al. "Entrapment of the suprascapular nerve". Journal of Bone Joint Surgery [Br] 81-B (1999): 414-419.

11. Bayam L., et al. "Pain mapping for common shoulder disorders". American Journal of Orthopadics (Belle Mead NJ) 40.7 (2011): 353-358.

12. Arm Squeeze. "Test: a new clinical test to distinguish neck from shoulder pain". European Spine Journal 22 (2013): 1558-1563.

13. Robert L McClain., et al. "Peripheral nerve blocks and postoperative physical therapy: a single-institution survey of physical therapists' preferences and opinions". Romanian Journal of Anaesthesia and Intensive Care 24.2 (2017): 115-124.

14. Tyagi A and Salhotra R. "Total hip arthroplasty and peripheral nerve blocks: Limited but salient role?”. Journal of Anaesthesiology, Clinical Pharmacology 34.3 (2018): 379-380.

15. Guay J., et al. "Nerve blocks or no nerve blocks for pain control after elective hip replacement (arthroplasty) surgery in adults". Cochrane Database of Systematic Reviews 10 (2017): CD011608. 
16. Detaille V., et al. "Use of continuous interscalene brachial plexus block and rehabilitation to treat complex regional pain syndrome of the shoulder". Annals of Physical and Rehabilitation Medicine 53 (2010): 406-416.

17. Rocourt Marianne HH., et al. "Evaluation of intratester and intertester reliability of the Constant-Murley shoulder assessment". Journal of Shoulder and Elbow Surgery 17.2 (2008): 364-369.

18. Constant CR and Murley AH. "A clinical method of functional assessment of the shoulder". Clinical Orthopaedics and Related Research 214 (1987): 160-164.

\section{Assets from publication with us}

- Prompt Acknowledgement after receiving the article

- Thorough Double blinded peer review

- Rapid Publication

- Issue of Publication Certificate

- High visibility of your Published work

Website: https://www.actascientific.com/

Submit Article: https://www.actascientific.com/submission.php

Email us: editor@actascientific.com

Contact us: +919182824667 